\title{
MODELO SOCCIO-COGNITIVO PARA A ESCOLHA DE CARREIRA: O PAPEL DA AUTO-EFICÁCIA E DE OUTRAS VARIÁVEIS RELEVANTES ${ }^{1}$
}

\section{Maiana Farias Oliveira Nunes Ana Paula Porto Noronha}

\section{RESUMO}

Esse artigo tem o objetivo de apresentar a Teoria Sócio-Cognitiva aplicada ao contexto do desenvolvimento de carreira e ilustrar suas aplicações em pesquisas realizadas em diferentes contextos. O foco do artigo residiu na explanação de variáveis importantes para a realização da escolha profissional, que auxiliam na motivação para a realização deste comportamento. Foram abordados os elementos pessoais, contextuais e de aprendizagem que auxiliam na formação das crenças de auto-eficácia e das expectativas de resultado que, conseqüentemente, influenciam a formação dos interesses profissionais, considerados elementos essenciais na motivação para a escolha de áreas profissionais específicas. Com base na revisão da literatura da área, verificou-se que esse referencial foi replicado em populações de diferentes etnias, de variadas fases do desenvolvimento vocacional, em estudos transversais e longitudinais, com pessoas de ambos os sexos e de níveis sócioeconômicos distintos. Desse modo, destaca-se a utilidade desde modelo para a compreensão da tomada de decisão no âmbito vocacional.

\section{PALAVRAS-CHAVE}

Teoria sócio-cognitiva do desenvolvimento de carreira; Escolha profissional; Auto-eficácia

\section{SOCIAL COGNITIVE THEORY OF CAREER DEVELOPMENT: THE ROLE OF SELF-EFFICACY AND OTHER IMPORTANT VARIABLES}

\begin{abstract}
This paper aims at introducing Social Cognitive Theory of Career Development and at illustrating its applications for studies conducted at different contexts. The main aspects which are important to make a vocational choice were highlighted, considering that they guide the motivation to this behavior. Personal, context and learning aspects that lead to self-efficacy beliefs formation and outcome expectations were pointed out, which, in turn, help the formation of vocational interests. All these aspects are considered essential for creating the motivation for choosing specific professional areas. According to the literature review, this approach was confirmed with people from different races, from varied moments of vocational development, in both longitudinal and cross-sectional studies, with people of both sexes and of different socio-economic levels. Therefore, the utility of this model is discussed, considering its importance for understanding people's motivation to make choices at the vocational realm.
\end{abstract}

\section{KEYWORDS}

Social cognitive theory of career development; Vocational choice; Self-efficacy

\footnotetext{
${ }^{1}$ As autoras agradecem a Coordenação de Aperfeiçoamento de Pessoal de Nível Superior- Capes.
} 
Esse artigo tem por objetivo apresentar a Teoria Sócio-Cognitiva do Desenvolvimento de Carreira (TSCDC), bem como algumas pesquisas que examinaram a sua adequação em diferentes contextos. Desse modo, serão abordados os principais elementos e concepções adotados por essa vertente, a fim de permitir ao leitor uma aproximação da teoria.

\section{A Teoria Sócio-Cognitiva do Desenvolvimento de Carreira (LENT; BROWN;} HACKETT, 1994) baseia-se em alguns pressupostos da Teoria Sócio-Cognitiva de Bandura (1986; 1997), que são aplicados no contexto da escolha acadêmica e profissional. Dentre os determinantes teóricos incorporados, originalmente expostos na Teoria Sócio-Cognitiva, Lent et al (1994) concordam com a idéia de que o homem baseia-se no pensamento auto-referente para a regulação da sua motivação e comportamento, mais especificamente na auto-eficácia e nas expectativas de resultado. A auto-eficácia refere-se à confiança na capacidade pessoal para organizar e executar atividades e certos cursos de ação, ou seja, à crença de que se é capaz de realizar ações específicas. Estas crenças podem variar desde aquelas mais favoráveis às mais desfavoráveis (BANDURA, 1986; 1997).

Por sua vez, ainda segundo esse autor, as expectativas de resultado refletem as expectativas sobre as conseqüências ou resultados das ações, também podendo ser positivas ou negativas. Juntamente com os pensamentos auto-referentes, esses autores destacam a importância de outros elementos na regulação do comportamento e motivação, tais como os aspectos afetivos, desenvolvimentais e biológicos dos indivíduos; ressaltando assim, a relação entre auto-eficácia e expectativas de resultados com variáveis pessoais e contextuais.

Considerando a visão geral da teoria proposta por Lent et al. (1994), é importante destacar que ela objetiva estudar grupos de adolescentes ou de pessoas na fase adulta inicial, uma vez que seu foco reside na escolha de carreira. Os autores afirmam que a auto-eficácia é um dos elementos que possui um papel central na escolha e no desenvolvimento de carreira. Ela é entendida como algo que ajuda a prever os interesses específicos dos alunos, seu desempenho escolar nas disciplinas preferidas, a gama de opções de carreira consideradas e a persistência e o sucesso obtido no campo escolhido. A partir disso, pode-se compreender que, nas áreas nas quais o sujeito possui crenças de auto-eficácia mais favoráveis, tenderá a apresentar expectativas de resultado positivas e, desse modo, isso poderá influenciar os comportamentos apresentados ou ainda as escolhas ocupacionais consideradas. 
De acordo com a TSCDC, alguns fatores antecedem a formação da auto-eficácia e das expectativas de resultado e, de maneira indireta, influenciam o processo de escolha. Esses aspectos possuem três eixos, quais sejam, pessoais, contextuais e experienciais, podendo funcionar como precursores das variáveis sócio-cognitivas ou como moderadores da relação teórica entre os construtos. Desse modo, a auto-eficácia é vista como um dos elementos que influencia as escolhas de carreira, mas não o único, uma vez que outros fatores também desempenham papel relevante na escolha de uma profissão, tal como já afirmado.

Sobre as variáveis pessoais, Lent et al. (1994) sintetizam que a escolha de uma carreira recebe inicialmente a influência das predisposições genéticas, do sexo, da etnia e das condição de saúde. No caso do sexo e etnia, destaca-se que não é a característica biológica em si que pode gerar diferenças nos comportamentos de escolha de carreira, mas sim, as reações advindas do ambiente social/cultural. Assim, ambos devem ser entendidos como fatores que influenciam a exposição seletiva a situações relevantes para a carreira. A título de exemplo, o fato de ser homem ou mulher pode influenciar no tipo de estímulo do ambiente que é oferecido desde a infância, nos "modelos" de profissionais que são mais frequentemente observados e nos comportamentos mais reforçados.

Ao lado disso, a condição de saúde (por exemplo, a existência de alguma doença crônica que restringe a locomoção de uma pessoa) pode influenciar a escolha de carreira de modo semelhante aos elementos anteriormente apresentados, no sentido da ocorrência de oportunidades e barreiras específicas a que as pessoas são expostas. Por fim, quanto à influência da genética na escolha de carreira, os autores formulam algumas hipóteses, porém não as aprofundam. Em linhas gerais, argumentam que as habilidades em potencial, em interação com o ambiente, podem parcialmente explicar a ligação entre a genética e os interesses profissionais e, posteriormente, a escolha.

Sobre a participação das condições contextuais ou o background, deve-se mencionar que elas são entendidas como as oportunidades (situações e comportamentos mais observados, tipo de modelo a que se tem acesso, dentre outras), os sistemas de suporte e barreiras (familiar, social, financeiro, emocional, entre outros) e as práticas de socialização específicas a que as pessoas são expostas ao longo do tempo. Outras variáveis contextuais mais abrangentes partilham de uma parcela de importância na escolha de carreira, como as 
condições financeiras e culturais do local onde se vive, por exemplo. Os fatores contextuais podem facilitar, restringir ou cancelar a volição na escolha de carreira. Os pesquisadores referidos enfatizam que os fatores contextuais moderam as relações entre interesses e objetivos profissionais; e entre os objetivos e a implementação da ação de escolha.

Assim, a relação entre interesses e objetivos por um lado, e entre objetivos e ação, por outro, será mais forte entre aqueles que percebem condições ambientais benéficas (poucas barreiras e muito suporte) e mais fraca nas pessoas que percebem condições menos favoráveis (muitas barreiras e pouco suporte). Além do poder moderador, os fatores contextuais também podem ter efeito direto sobre a escolha de carreira. Por exemplo, condições sócio-econômicas e educacionais que ampliam as oportunidades permitem mais facilmente que os interesses sejam transformados em objetivos de escolha correspondentes.

No que tange às oportunidades, indica-se, por exemplo, que o acesso a ambientes educacionais específicos pode influenciar a qualidade e o tipo de experiências de aprendizagem que alguém recebe, o que tende a influenciar na seleção de determinadas atividades ocupacionais. Em outras palavras, processos de socialização diferenciados ou oportunidades específicas tendem a promover o desenvolvimento de habilidades, interesses e padrões de auto-eficácia em áreas específicas. As oportunidades podem ser divididas entre as percebidas e as concretamente existentes (objetivas), sendo que ambas são importantes para o desenvolvimento de carreira. A inclusão da percepção do ambiente de maneira subjetiva valoriza os aspectos cognitivos da Teoria Sócio Cognitiva de Desenvolvimento de Carreira, uma vez que se trata de uma interpretação das condições objetivas vivenciadas (LENT et al., 1994).

Adicionalmente, esses autores dividem a estrutura de oportunidades em outros dois grupos, que se referem ao "momento" de influência desses elementos. O primeiro relaciona-se às oportunidades mais distantes temporalmente do momento de escolha, ou seja, as influências que precedem e ajudam a moldar interesses e auto-cognições, tais como oportunidades diferenciais para a realização de certas atividades, exposição a certos modelos, suporte emocional e financeiro para participar de certas atividades e o processo de socialização do papel cultural e de sexo das pessoas. O segundo grupo, por sua vez, trata das influências temporalmente mais próximas do momento de escolha de carreira, como contatos 
de trabalho (network) e barreiras estruturais (por exemplo, práticas de seleção preconceituosas). No entanto, esses dois tipos de influência não ocorrem separadamente; na prática, eles se misturam, pois alguns fatores estão sempre presentes (como, por exemplo, a família) e podem ter influência durante todo o processo de desenvolvimento de carreira, apesar do peso dado a esses fatores poder variar ao longo do tempo.

Com uma argumentação semelhante, Bandura (2006) afirma que um dos elementos que operam indiretamente nas trajetórias ocupacionais dos adolescentes é o status sócio-econômico familiar. Acredita-se que este fator atue por meio da elevação das aspirações educacionais dos pais em relação ao desempenho escolar dos filhos e pela crença de que podem estimular o desenvolvimento acadêmico destes. Mais especificamente, quanto mais os pais acreditam que podem estimular os filhos, maiores as aspirações sobre o futuro educacional/ocupacional e mais os pais agem de modo a auxiliar na construção da autoeficácia acadêmica, social e auto-regulatória dos filhos. Essa sequiência de eventos, desenvolvidos ao longo do tempo, tende a promover melhores resultados acadêmicos para os jovens.

No que se refere aos aspectos experienciais ou de aprendizagem, esses são descritos como as fontes de informação de auto-eficácia e de expectativas de resultado. Nesse sentido, foram descritas quatro fontes de auto-eficácia, quais sejam, a experiência pessoal, a aprendizagem vicária, a persuasão verbal e os indicadores fisiológicos. A experiência pessoal provê indicadores diretos de competência por meio do sucesso na realização das ações; a aprendizagem vicária envolve a percepção de que, se outros semelhantes são capazes de realizar certas atividades, a pessoa em questão também poderá executá-la, desde que ela se veja num mesmo "nível" de habilidade que o sujeito observado; a persuasão verbal ocorre por meio de feedbacks, podendo ajudar a reforçar ou a enfraquecer a crença na capacidade pessoal; e os indicadores fisiológicos são os sinais físicos usados como meio para avaliar a capacidade ou disfunção em uma dada atividade, tais como um humor favorável ou a ansiedade (BANDURA, 1977; 1986; 1997). Assim, a ocorrência de sucessos repetidos em certas atividades, o sucesso de outros considerados semelhantes em tarefas específicas, a expressão de encorajamentos e a avaliação de sinais físicos como "força" para determinada atividade tendem a aumentar a confiança na capacidade pessoal para realização da atividade. As fontes interagem na formação da auto-eficácia e possuem utilidade a partir da 
interpretação que se dá aos eventos, e não pelos eventos em si. A título de exemplo, pode-se supor que mesmo quando alguém tem sucesso na realização de uma atividade, ela foque nos aspectos negativos do seu desempenho e, desse modo, mantenha uma crença desfavorável de auto-eficácia relacionada àquele domínio.

Concluída a explicação dos fatores que antecedem a auto-eficácia e as expectativas de resultado, conforme o modelo proposto por Lent et al. (1994) para a escolha de carreira, faz-se importante discorrer sobre esses fatores mais detalhadamente. A autoeficácia é relevante nas tomadas de decisão, pois, a partir dessas crenças, as pessoas têm bases para realizar as escolhas diárias sobre quais atividades realizarão e quanto tempo e esforço empenharão com cada tarefa ou objetivo. Mais detalhadamente, a auto-eficácia influencia se a pessoa pensa de forma pessimista ou otimista com relação a certos objetivos, uma vez que há uma tendência das pessoas serem mais otimistas nas atividades que possuem confiança da sua capacidade (BANDURA, 2001). Por ser uma variável que deve influenciar a escolha dos comportamentos que são colocados em prática, a auto-eficácia é um componente importante para o desenvolvimento pessoal, para as adaptações às situações de vida e para as mudanças pessoais. As atividades que as pessoas escolhem realizar geram um impacto maior na vida delas, ao promover o desenvolvimento de certas habilidades, de estilos de vida e de valores específicos (BANDURA, 2006).

Sobre o papel que a auto-eficácia possui na definição das ações que as pessoas optam por realizar, convém destacar que, de uma maneira geral, tende-se a evitar tarefas e situações que se acredita exceder a capacidade e, por outro lado, provavelmente escolhe-se alcançar e realiza-se com segurança as atividades que se julgam capazes de executar. As crenças favoráveis de auto-eficácia tendem a facilitar o desenvolvimento das potencialidades das pessoas, pois elas se esforçam para a realização das ações. Mais especificamente, mesmo quando as pessoas ainda não possuem certa habilidade, o esforço sustentado (gerado por crenças de auto-eficácia favoráveis) acaba levando ao desenvolvimento do potencial. Por outro lado, quando há crenças desfavoráveis de auto-eficácia, as pessoas evitam certos ambientes e atividades, o que tende a retardar o desenvolvimento de suas competências na atividade em questão (BANDURA, 1986; 1997). 
Betz e Borgen (2000), com uma proposta teórica consoante com a de Lent et al. (1994), afirmam que a auto-eficácia é um mediador do comportamento de escolha, que pode ser observado por meio de três indicadores: comportamentos de aproximação versus evitação de atividades ocupacionais; qualidade do desempenho no comportamento; e persistência em face de obstáculos ou experiências que não confirmam as crenças. Ainda, vale destacar que o comportamento de aproximação é especialmente importante no contexto de decisão de carreira, pois se refere às atividades, cursos ou ocupações que o sujeito quer tentar ou irá perseguir. Por outro lado, as ações de evitação provavelmente ocorrem quando há crenças desfavoráveis de auto-eficácia, que levariam à eliminação de opções de carreira. Além disso, os autores salientam o efeito indireto da auto-eficácia na escolha de carreira, por meio da limitação do desenvolvimento dos interesses, já que, em alguns casos, podem gerar a evitação de novas experiências e oportunidades de aprendizagem.

Por sua vez, de acordo com Lent et al. (1994), como já afirmado, as expectativas de resultado são as crenças sobre as consequiências observáveis e não observáveis das ações, ou seja, são as crenças sobre os resultados prováveis das ações, que podem referir-se a aspectos físicos (recompensas financeiras), sociais (aprovação de colegas ou familiares) ou de auto-avaliação (satisfação pessoal). Conforme já mencionado, a associação entre auto-eficácia favorável e expectativas de resultado positivas tende a aumentar a probabilidade de escolha de uma área profissional. Apesar de existir uma relação entre a auto-eficácia e as expectativas de resultados, não há uma associação linear, ou seja, nem sempre uma auto-eficácia favorável será acompanhada por expectativas de resultado favoráveis. Isso pode ocorrer quando, por exemplo, uma pessoa que possui crença favorável de auto-eficácia para a escrita, simultaneamente apresentar baixa expectativa de resultado, na medida em que considera que o mercado de trabalho para escritores apresenta um grande índice de desemprego.

Os interesses profissionais, por sua vez, são compreendidos como padrões de gostos, aversões e indiferenças acerca de atividades e ocupações relacionadas a uma carreira, e seu desenvolvimento se dá ao longo dos anos escolares. Supõe-se que eles influenciem mais diretamente a escolha de carreira, porém interagindo com fatores externos como a pressão social e os fatores econômicos, que, por sua vez, influenciam o nível (cursar ensino médio, técnico ou superior) e o conteúdo das escolhas realizadas, ou seja, o tipo de atividade que a pessoa escolhe realizar profissionalmente. Desse modo, uma pessoa pode escolher sair do 
Ensino Médio e trabalhar imediatamente, sem dar continuidade aos estudos; pode fazer um curso técnico e posteriormente ingressar no mercado de trabalho; pode fazer curso do Ensino Superior e pós-graduações, melhorando sua qualificação profissional, porém aumentando o tempo que o distancia de ingressar no mundo do trabalho. Assim, se alguém precisa de um retorno financeiro imediato, é provável que comece a trabalhar ainda no Ensino Médio e que não estenda os estudos; em outros casos, em que houver apoio financeiro familiar, por exemplo, a pessoa ficará mais livre para escolher diferentes caminhos profissionais (LENT et al., 1994).

Quanto ao o desenvolvimento dos interesses, os autores afirmam que ao longo do tempo (na infância e adolescência), as pessoas são expostas a uma série de atividades potencialmente relevantes para a carreira. Além disso, também observam ou ouvem sobre performances de pessoas variadas em certas ocupações, do mesmo modo que são reforçadas por realizar certas atividades (entre muitas outras possíveis) e por atingir uma performance satisfatória na atividade escolhida. Por meio do envolvimento repetido com certas atividades, da aprendizagem por observação e o recebimento de feedback de pessoas significativas em seu percurso de vida, as pessoas continuamente refinam suas habilidades, formando suas crenças de auto-eficácia e expectativas de resultado.

Em síntese, pode-se afirmar que as crenças e as expectativas de resultados influenciam fortemente a formação dos interesses profissionais. De tal modo, é pouco provável que um forte interesse surja em áreas em que há crença desfavorável de auto-eficácia ou em que há expectativas de resultado negativas ou neutras. Para Lent et al. (1994), as crenças de auto-eficácia, aliadas às expectativas de resultados, precedem a formação dos interesses. Crenças favoráveis de auto-eficácia e expectativa positiva acerca das recompensas futuras tendem a gerar interesses por atividades específicas e estimulam intenções do sujeito em continuar se comprometendo com elas. Por outro lado, aquelas situações vinculadas a crenças de auto-eficácia e expectativas de resultado desfavoráveis ou negativas devem provocar desgosto e evitação da atividade, gerando exclusões das opções de carreira ligadas a essas crenças. Apesar de reconhecer uma relação que tende a ser positiva entre auto-eficácia e interesses profissionais, Bandura (1986) propôs que pode haver um atraso temporal entre crenças de auto-eficácia recentemente adquiridas e o desenvolvimento do interesse por atividades antes vistas como neutras ou não prazerosas para a pessoa. Desse modo, o 
crescimento do interesse não pode ser visto como uma conseqüência imediata das crenças de auto-eficácia.

Na seqüência do modelo de escolha de carreira, de acordo com Lent et al. (1994), o desenvolvimento de interesse por certas atividades favorece a formação de certos objetivos, que tendem a aumentar a probabilidade da seleção e prática da atividade específica. Os objetivos ou metas podem ser definidos como a determinação para engajar-se em uma atividade particular ou para alcançar um resultado futuro com a ação. Por meio deles, as pessoas podem organizar e orientar o seu comportamento por longos períodos, mesmo quando não recebem reforços externos, e ainda aumentar a probabilidade de que as consequiências desejadas sejam atingidas.

Nesse ponto, há que se destacar uma distinção entre as intenções de escolha (objetivos) e as escolhas realizadas. Uma pessoa pode ter intenções de escolha que, em função de outras variáveis, como, por exemplo, as contextuais, podem não ser concretizadas. Convém lembrar que as escolhas efetuadas não são ações congeladas, elas também passam por revisões em função dos acontecimentos novos e dos resultados das performances. Por exemplo, alguém pode entrar num curso de Engenharia porque possuía interesse na área, porém, ao ter resultados ruins nas disciplinas de física, poderá mudar os objetivos anteriores e mudar a opção realizada. A concepção dos objetivos refere-se à intenção de realizar ações ou um conjunto de ações específicas (exemplos: tornar-se um engenheiro; fazer um curso de psicologia) e não ao nível de performance alcançado com a ação. Desse modo, há uma ênfase no conteúdo ou na direção das opções relacionadas à carreira e não na qualidade dos desempenhos obtidos em certas áreas.

O elemento seguinte da TSCDC refere-se ao envolvimento ou prática de certas atividades, que tende a gerar resultados específicos (sucessos ou fracassos), e possibilitam a revisão das crenças de auto-eficácia e das expectativas de resultado. Desse modo, ilustra-se a retro-alimentação que pode ocorrer entre os construtos e o caráter dinâmico desta proposta teórica. Entende-se que esse processo ocorre continuamente ao longo da vida, porém o mesmo é mais fluido até o final da adolescência. Após a cristalização dos interesses, apenas situações extremas poderão gerar a revisão desses elementos, tais como a perda de emprego, aposentadoria e outras situações de transição de carreira. Analisando o modelo de escolha de 
carreira como um todo, os autores indicam ainda que, em qualquer momento, certas variáveis poderão ter diferentes pesos (LENT et al., 1994).

\section{APLICAÇÕES EM PESQUISA DA TEORIA SÓCIO-COGNITIVA DO DESENVOLVIMENTO DE CARREIRA (TSCDC)}

O modelo da TSCDC tem sido testado e refinado por vários pesquisadores, em diferentes contextos. Algumas pesquisas serão apresentadas, a seguir, em ordem cronológica, de modo a demonstrar a evolução do estudo destes construtos ao longo do tempo.

O estudo de Fouad e Smith (1996), com 380 alunos do Ensino Fundamental, deu suporte às argumentações de que os interesses são reflexo da auto-eficácia, juntamente com as expectativas de resultado e, que a auto-eficácia afeta as expectativas de resultados, os objetivos e ações de escolha. Nesse estudo, ao testar as variáveis sexo e idade em modelos de equações estruturais, a idade só apresentou contribuição significativa para os interesses profissionais, enquanto o sexo o fez para interesses e expectativas de resultados. Essas duas variáveis não contribuíram significativamente para a auto-eficácia ou para os outros elementos do modelo, conforme previsto por Lent et al. (1994).

Resultado semelhante sobre a relação entre interesses, auto-eficácia e expectativas de resultado foi verificado com 164 universitários negros do $1^{\circ}$ ano de curso, de ambos os sexos, de cursos variados, sendo que a maioria estava na área de Ciências Naturais (52\%) ou Ciências Sociais (41\%). A variável 'atitudes relacionadas à identidade racial', que refere-se a um senso de identidade baseado na percepção de que se possui características comuns a um grupo racial específico, teve pequeno efeito no modelo como um todo, sugerindo que ela não tem influência tão forte sobre o modelo como outras variáveis pessoais, a exemplo do sexo (GAINOR; LENT, 1998).

Outro resultado favorável foi encontrado por Smith (2002), na análise das respostas de 278 universitários, considerando especificamente o campo da tecnologia e informática. A autora verificou que apenas as fontes de auto-eficácia denominadas experiência pessoal e indicadores fisiológicos previram significativamente os interesses por 
tecnologia de informação, do mesmo modo que a auto-eficácia e as expectativas de resultado o fizeram com relação a esse mesmo tipo de interesse.

A investigação de Flores e O’Brien (2002) testou e ampliou aspectos desse modelo, com a participação de 364 adolescentes México-Americanos do sexo feminino. Nesse estudo, houve suporte a alguns aspectos do modelo da TSCDC. Por exemplo, a autoeficácia para atividades consideradas como tradicionalmente masculinas previu interesses por estas mesmas atividades, o prestígio e o nível de tradicionalismo associado à carreira (se ocupavam profissões predominantemente desempenhadas por homens ou por mulheres). De modo semelhante, a percepção de suporte e de poucas barreiras tiveram um impacto significativamente positivo nas aspirações e escolhas profissionais. Outro dado interessante é que essas adolescentes escolheram profissões de mais prestígio baseadas na tentativa de aprovação dos outros e de atender as expectativas familiares. Com base nesse resultado, as autoras destacaram que, em culturas como a mexicana, em que o papel da família é muito importante no processo de escolha da profissão, a questão da origem cultural familiar deve ser explorada em maior detalhe.

Nessa mesma pesquisa, alguns resultados não ocorreram conforme era esperado, a partir da análise da proposta de Lent et al (1994). Um exemplo que refuta o proposto no modelo é que a atitude feminista, elemento que, segundo as autoras, deveria preceder a formação das crenças de auto-eficácia, não teve contribuição significativa na previsão desse construto. Outro dado não esperado foi que o nível educacional das mães das alunas e o grau de tradicionalismo nas ocupações delas não influenciou a auto-eficácia para que as filhas seguissem ocupações que não eram tradicionalmente femininas. Sobre esse resultado, as autoras sugeriram que outras pesquisas deveriam avaliar a influência do nível educacional e tradicionalismo da ocupação paterna e de outros membros da família, pois os dados apenas sobre a mãe não pareceram suficientes. Por fim, não se encontrou o resultado esperado nas relações entre auto-eficácia e aspirações de carreira e entre interesses e objetivos de carreira. As autoras argumentam que talvez as alunas de origem mexicanas avaliadas não pudessem escolher com base em seus interesses ou auto-eficácia, mas sim devessem escolher em função de outras variáveis contextuais. Mais especialmente, talvez em famílias mais conservadoras as jovens não tenham suporte para escolher carreiras não tradicionais. 
Por sua vez, a pesquisa de Lent, Brown, Nota e Soresi (2003), com 796 alunos italianos do Ensino Médio, verificou resultados favoráveis à replicação do modelo da Teoria Sócio-Cognitiva para o Desenvolvimento de Carreira (TSCDC) sobre a relação entre interesses, auto-eficácia, expectativas de resultado e objetivos de escolha. Contudo, neste trabalho, a percepção de barreiras e suporte atuou apenas indiretamente sobre as considerações sobre a escolha, por meio da auto-eficácia, e não diretamente, conforme previsto pelo modelo da TSCDC.

Já o estudo longitudinal realizado com 204 mulheres, que no primeiro momento da coleta de dados estavam no Ensino Médio e foram contatadas novamente quatro anos depois, evidenciou que boa parte do modelo proposto pela TSCDC foi replicado (NAUTA; EPPERSON, 2003). Houve um dado interessante, contudo, quando interesses, auto-eficácia e escolhas de carreira foram analisados: o contexto das Ciências foi representado por um modelo estatisticamente significativo, porém nos cursos de Tecnologia, o mesmo não ocorreu. Os autores sugerem, ao discutir os resultados, que provavelmente os instrumentos usados não apresentaram itens adequados para representar os interesses por Tecnologia, o que, no entanto, não geraria dúvidas sobre a aplicabilidade do modelo.

Em direção semelhante, a relação entre auto-eficácia, expectativas de resultado e a escolha concretamente realizada foi atestada em um estudo com 225 universitários. Eles responderam escalas sobre a auto-eficácia e expectativas de resultado para atividades ocupacionais específicas. Verificou-se forte relação entre os construtos e a escolha concretamente realizada (LINDLEY, 2005).

De modo complementar, a pesquisa de Lent et al. (2005) sugere que o modelo da TSCDC produz bons índices de ajuste entre os diferentes sexos e entre diferentes tipos de universidade (predominantemente freqüentadas por brancos ou negros). Nessa investigação, participaram 487 estudantes de cursos de engenharia de três instituições diferentes. Foram avaliadas as relações entre auto-eficácia, expectativas de resultados, interesses pela área técnica, suporte e barreiras sociais e objetivos educacionais. Os autores argumentam que a utilidade preditiva do modelo é tão boa para homens como para mulheres, do mesmo modo que para estudantes de diferentes universidades. Destaca-se, ainda, que esse tipo de estudo serve para desmistificar a área da engenharia como algo "não tradicional" para as mulheres 
americanas, uma vez que elas possuíam auto-eficácia e interesses comparáveis com os homens, porém elas acreditam possuir maior suporte e menos barreiras que os homens.

Por sua vez, a pesquisa de Cunningham, Bruening, Sartore, Sagas e Fink (2005) também atestou a utilidade do modelo da TSCDC com 197 universitários de cursos de esporte e lazer. Um dado relevante verificado foi que a percepção de suporte e barreiras atua indiretamente sobre as intenções de escolha, por meio da auto-eficácia. Os autores sugerem, desse modo, uma alteração para as direções propostas no modelo de Lent et al. (1994), no sentido de que as variáveis contextuais proximais do modelo não devem ser vistas como elementos que possuem impacto direto sobre a escolha, mas sim indireto, por meio da autoeficácia.

O estudo realizado com 302 adolescentes do sexo masculino de origem MéxicoAmericana também acrescentou informações ao modelo da TSCDC (FLORES et al., 2006). A auto-eficácia para carreiras não tradicionais foi prevista pelo nível de aculturação e suporte parental. Ainda, a auto-eficácia para carreiras não tradicionais previu o interesse por carreiras não tradicionais, enquanto a escolha expressa de carreiras não tradicionais foi prevista pelos interesses não tradicionais e pelo não tradicionalismo das carreiras dos genitores masculinos. Com isso, destacaram-se variáveis contextuais não salientadas inicialmente no modelo de Lent et al. (1994), tais como o nível de aculturação dos participantes e o tradicionalismo relacionado às carreiras.

Outra questão que pode ser levantada é se as considerações da TSCDC também são aplicáveis a populações consideradas em risco psicossocial. Com esse intuito, Jackson, Potere e Brobst (2006) analisaram as respostas de 93 alunos do Ensino Fundamental de uma escola que atendia famílias de baixa renda. Os autores encontraram que as experiências de aprendizagem estavam positiva e significativamente associadas com os interesses e, por sua vez, a auto-eficácia para atividades ocupacionais estava correlacionada com os interesses. No entanto, as experiências de aprendizagem não se associaram significativamente com a ocupação ideal. Esse resultado foi interpretado pelos autores como algo provavelmente decorrente dos jovens sonharem com uma ocupação que acreditavam estar além de suas capacidades. 
Com objetivo de investigar os pressupostos da TSCDC em uma população culturalmente diversa, Ali e McWhirter (2006) analisaram informações sobre 338 adolescentes do Ensino Médio oriundos de região rural, residentes nas montanhas do Apalache. Os jovens tiveram suas aspirações sobre o quê fazer após a conclusão do Ensino Médio classificadas em quatro grupos, a saber, trabalhar com dedicação exclusiva, fazer um curso técnico, fazer um curso de bacharelado ou fazer um curso com bacharelado e que permita atuação profissional.

Os autores verificaram que a auto-eficácia vocacional/educacional, as expectativas de resultado, a percepção de barreiras e o status sócio-econômico foram fatores que contribuíram significativamente para prever o caminho escolhido a ser percorrido após a conclusão do Ensino Médio. Os jovens de menor nível sócio-econômico tiveram auto-eficácia e expectativas de resultados mais baixas; percebiam mais barreiras e pretendiam trabalhar imediatamente após a saída da escola, enquanto os de nível sócio-econômico mais alto tiveram auto-eficácia e expectativas de resultado mais altas; percebiam menos barreiras e desejavam fazer um curso de bacharelado ou profissional. Os autores discutiram que, quando da avaliação de conceitos da TSCDC, é importante levar em consideração a situação da amostra e seu nível sócio-econômico, uma vez que as ocupações profissionais abordadas em intervenções vocacionais devem ser culturalmente consistentes com o grupo alvo. No caso específico do grupo analisado, as condições sociais e culturais da população que habitava nas montanhas do Apalache não favoreciam a aspiração de algumas profissões, a menos que fosse feita a opção de emigrar da região.

Considerando uma área profissional de destaque atualmente em função das mudanças climáticas e do aquecimento global, o estudo de Quimby, Wolfson e Seyala (2007) abordou o domínio das Ciências Ambientais. Nesse caso, a pesquisa realizada com 132 adolescentes afro-americanos encontrou resultados favoráveis ao modelo da TSCDC, em que a auto-eficácia investigativa, as expectativas de resultado, as barreiras e suporte percebidos e a preocupação com o meio ambiente previram os interesses por Ciências Ambientais.

A seu turno, Teixeira (2007) relatou uma síntese das pesquisas sobre auto-eficácia realizadas em Portugal. Sobre a testagem do modelo da Teoria Sócio-Cognitiva para o desenvolvimento de Carreira, no que se refere ao desenvolvimento dos interesses e à 
realização de escolhas vocacionais, as investigações consultadas pela autora sugeriram a adequação deste modelo à realidade portuguesa. Os artigos encontrados também indicaram que a auto-eficácia e o desempenho escolar, conjuntamente, contribuem na decisão do tipo de curso a ser seguido na saída do Ensino Médio e no nível dos projetos, ou seja, se a pessoa pretende começar a trabalhar imediatamente após a conclusão dos estudos ou fazer curso superior, entre outros. A autora acrescentou que a auto-eficácia associa-se a crenças motivacionais mais profundas, que auxiliam a compreender o modo que as pessoas representam as carreiras para si.

Vieira e Coimbra (2008) analisaram as associações entre auto-eficácia na transição para o trabalho, auto-eficácia na procura de trabalho, expectativas de resultado da procura de trabalho e objetivos de investimento profissional em 245 universitários portugueses, do último ano de curso. A correlação entre a auto-eficácia para a transição para o mercado de trabalho teve índices significativos e positivos com os três construtos. Os autores interpretaram que os dois tipos de escalas de auto-eficácia (para a transição e para a procura de trabalho) apresentaram validade convergente $(r=0,72)$, além de indicarem a coerência com as formulações teóricas da Sócio-cognitiva, no que diz respeito à relação da auto-eficácia com expectativas de resultado $(r=0,47)$ e objetivos de carreira $(r=0,32)$. Concluiu-se sobre a adequação na análise do modelo da Teoria Sócio-Cognitiva na realidade portuguesa, considerando o contexto da transição de universitários para o mercado de trabalho.

Os estudos de Nunes e Noronha (2008) e de Nunes (2009) realizados no Brasil, com público adolescente, confirmaram as expectativas de associações significativas entre auto-eficácia e interesses. Mais especificamente, houve correlações de fracas a moderadas entre os construtos, o que levou ao questionamento sobre a existência de sobreposições entre a auto-eficácia e os interesses profissionais em áreas profissionais específicas (NUNES, 2009). Desse modo, trata-se de outro achado que sugere a importância do contínuo aprimoramento da TSCDC, de modo a testá-lo em diferentes realidades sócio-culturais. 


\section{CONSIDERAÇÕES FINAIS}

O presente artigo pretendeu apresentar os elementos inerentes à Teoria SócioCognitiva no contexto do desenvolvimento de carreira, bem como discutir os achados de investigações realizadas em diferentes contextos. A justificativa para essa elaboração se deu em razão do pequeno escopo relacionado ao tema, no Brasil. Em conjunto, as pesquisas relacionadas auxiliam na visualização do desenvolvimento teórico e empírico da TSCDC no âmbito internacional, porém, no Brasil, há necessidade de investimento de pesquisas com essa abordagem, uma vez que poucas referências puderam ser recuperadas sobre o tema em publicações nacionais (NUNES, 2008). Ainda que a maior parte dos estudos tenha confirmado a teoria, alguns estudos salientaram o detalhamento de outros aspectos, inicialmente não explicitados por Lent et al. (1994), como a pesquisa de Flores e O’Brien (2002) e de Flores et al. (2006), que acrescentaram aspectos à análise dos elementos de background contextual, como as atitudes feministas, o nível de aculturação e o grau de tradicionalismo relacionado às carreiras.

Contudo, a maioria das pesquisas confirmou as expectativas teóricas sobre a relação entre interesses, auto-eficácia e expectativas de resultado, sendo essa sempre positiva (FOUAD; SMITH, 1996; GAINOR; LENT, 1998; SMITH, 2002; LENT et al., 2003; NAUTA; EPPERSON, 2003; LINDLEY, 2005; LENT et al., 2005; CUNNINGHAM et al., 2005; JACKSON et al., 2006; ALI; MCWHIRTER, 2006; QUIMBY et al., 2007; TEIXEIRA, 2007; VIEIRA; COIMBRA, 2008; NUNES; NORONHA, 2008; NUNES, 2009). Assim, em geral, as pesquisas confirmaram que crenças de auto-eficácia favoráveis e expectativas de resultado positivas tenderam a ser acompanhadas por interesses profissionais em áreas correspondentes, o que apresentou impacto para a investigação dos caminhos escolhidos na concretização das escolhas profissionais. Em outras palavras, pessoas com auto-eficácia favorável para atividades de Artes, por exemplo, e que também apresentam expectativas de resultado positivas quanto ao trabalho nessa área tenderão a apresentar interesses profissionais por Artes. 
Em acréscimo, foi possível replicar a teoria em populações de diferentes etnias, dentre eles, americanos, afro-americanos, afro-mexicanos, italianos, portugueses, brasileiros, de diferentes fases do desenvolvimento vocacional (do Ensino Fundamental ao Superior), em estudos transversais e longitudinais, com pessoas de ambos os sexos e de diferentes níveis sócio-econômicos. É possível perceber, portanto, a robustez das proposições do modelo da TSCDC e a grande atratividade que vem conquistando entre diferentes pesquisadores. Como conseqüência, pode-se ter maior confiança para o uso desse modelo para a compreensão das motivações para a tomada de decisão no âmbito vocacional.

Destarte, espera-se que o presente trabalho contribua com o avanço deste domínio no contexto do desenvolvimento de carreira brasileiro, de modo que mais profissionais façam uso do modelo e que mais pesquisadores se dediquem à busca de novos achados. Ao lado disso, faz-se necessário salientar que os estudos nacionais poderão gerar um escopo mais apropriado às idiossincrasias e vicissitudes aqui encontradas. Assim, acredita-se, a autoeficácia e os demais elementos da TSCDC poderão ser mais facilmente identificados e compreendidos.

\section{REFERÊNCIAS}

ALI, S. R.; MCWHIRTER, E. H. Rural Appalachian youth's vocational/educational postsecondary aspirations: applying social cognitive Career Theory. Journal of Career Development, v. 33, n. 2, p. 87-111, 2006.

BANDURA, A. Adolescent development from an Agentic perspective In: PAJARES, F.; URDAN, T. (Ed.). Self-efficacy of adolescents. Greenwich: Information Age Publishing, 2006.

Self-efficacy: the exercise of control. New York: W. H. Freeman and Company, 1997.

Self-efficacy: toward a unifying theory of Behavioral Change. Psychological

Review, v. 84, n. 2, p. 191-215, 1977.

Social cognitive theory: an Agentic perspective. Annual Review of Psychology, v. 52, n. 1, p. 1-26, 2001. 
Social foundations of thought and action: a social cognitive theory. Englewood Cliffs, NJ: Prentice-Hall, 1986.

BETZ, N. E.; BORGEN, F. H. The future of career assessment: integrating vocational interests with self-efficacy and personal styles. Journal of Career Assessment, v. 8, n. 4, p. 329-338, 2000.

CUNNINGHAM, G. B. et al. The application of social cognitive career theory to sport and leisure career choices. Journal of Career Development, v. 32, n. 2, p. 122-138, 2005.

FLORES, L. Y. et al. Testing a model of nontraditional career choice goals with Mexican American adolescent men. Journal of Career Assessment, v. 14, n. 2, p. 214-234, 2006.

.; O'BRIEN, K. M. The career development of Mexican American adolescent women: a test of social cognitive career theory. Journal of Counseling Psychology, v. 49, n. 1, p. 14$27,2002$.

FOUAD, N. A.; SMITH, P. L. A test of a social cognitive model for middle school students: math and science. Journal of Counseling Psychology, v. 43, n. 3, p. 338-346, 1996.

GAINOR, K. A.; LENT, R. Social cognitive expectations and racial identity attitudes in predicting the math choice intentions of Black College Studentes. Journal of Counseling Psychology, v. 45, n. 4, p. 400-413, 1998.

JACKSON, M. A.; POTERE, J. C.; BROBST, K. A. Are success learning experiences and self-efficacy beliefs associated with occupational interests and aspirations of at-risk urban youth? Journal of Career Assessment, v. 14, n. 3, p. 333-353, 2006.

LENT, R. et al. Social cognitive predictors of Academic Interests and goals in engineering: utility for women and students at historically black universities. Journal of Counseling Psychology, v. 52, n. 1, p. 84-92, 2005.

et al. Testing social cognitive interest and choice hypotheses across Holland types in Italian high school students. Journal of Vocational Behavior, v. 62, n. p. 101-118, 2003.

LENT, R.; BROWN, S. D.; HACKETT, G. Toward a unifying social cognitive theory of career and academic interest, choice and performance. Journal of Vocational Behavior, v. 45 , n. p. 79-122, 1994.

LINDLEY, L. D. Perceived barriers to career development in the context of social cognitive career theory. Journal of Career Assessment, v. 13, n. 3, p. 271-287, 2005.

NAUTA, M. M.; EPPERSON, D. L. A Longitudinal examination of the social-cognitive model applied to high school girls' choices of nontraditional college majors and aspirations. Journal of Counseling Psychology, v. 50, n. 4, p. 448-457, 2003. 
NUNES, M. F. O. Funcionamento e desenvolvimento das crenças de auto-eficácia: uma revisão. Revista Brasileira de Orientação Profissional, Ribeirão Preto, v. 9, n. 1, p. 29-42, 2008.

NUNES, M. F. O.; NORONHA, A. P. Análise correlacional entre interesses e auto-eficácia para atividades ocupacionais. In: IV CONFERÊNCIA DESENVOLVIMENTO

VOCACIONAL/I VIRTUAL: INVESTIGAÇÃO E ENSINO, 2008, Braga. Anais... Braga: [s.n.], 2008.

NUNES, M. F. O.; NORONHA, A. P. Estudos psicométricos da escala de auto-eficácia para atividades ocupacionais. 2009. Tese (Doutorado em Psicologia) - Universidade São Francisco, Itatiba, 2009.

QUIMBY, J. L.; WOLFSON, J. L.; SEYALA, N. D. Social cognitive predictors of African American adolescents' career interests. Journal of Career Development, v. 33, n. 4, p. 376394, 2007.

SMITH, S. M. Using the social cognitive model to explain vocational interest in information technology. Information technology, learning and performance Journal, v. 20, n. 1, p. 1-9, 2002.

TEIXEIRA, M. O. As crenças de eficácia académica na formação dos interesses e das escolhas vocacionais. Psychologica, v. 44, n. p. 11-23, 2007.

VIEIRA, D.; COIMBRA, J. L. Auto-eficácia na transição para o trabalho: contributos para a validação de um instrumento. In: IV CONFERÊNCIA DE DESENVOLVIMENTO

VOCACIONAL/ I CONFERÊNCIA VIRTUAL: INVESTIGAÇÃO E ENSINO, 2008, Braga. Anais... Braga: [s.n.], 2008. 
Psicóloga pela Faculdade Ruy Barbosa, mestre e doutorada em Psicologia com ênfase em Avaliação Psicológica pelo Programa de Pós-graduação

Stricto Sensu em Psicologia da Universidade São Francisco. Tem experiência com os temas 'Construção e Validação de Instrumentos Psicológicos', 'Orientação Profissional' e 'Avaliação da Personalidade'. Email: maiananunes@mac.com

ANA PAULA PORTO NORONHA

Psicóloga, mestre e doutorada em Psicologia Ciência e Profissão pela Pontifícia Universidade Católica de Campinas (1999). Atualmente é professora associada do Programa de Pós-graduação Stricto Sensu em Psicologia da Universidade São Francisco. Tem experiência na área de Psicologia, com ênfase em Fundamentos e Medidas da Psicologia. É bolsista produtividade em pesquisa do CNPq. Email: ana.noronha@saofrancisco.edu.br

Recebido em: 20/07/2009

Publicado em: 30/10/2009 